\title{
STEREOLOGY OF NEURONAL CONNECTIONS (MYELINATED FIBERS OF WHITE MATTER AND SYNAPSES OF NEOCORTEX) IN HUMAN BRAIN
}

\author{
YONG TANG ${ }^{\#}$, JENS R NYENGAARD ${ }^{1}$, BENTE PAKKENBERG ${ }^{2}$ AND HANS JøRGEN G GUNDERSEN ${ }^{1}$ \\ ${ }^{1}$ Stereological Research Laboratory and Electron Microscopy Laboratory, University Institute of Pathology, \\ Institute of Experimental Clinical Research, University of Aarhus, ${ }^{2}$ Research Laboratory for Stereology and \\ Neuroscience, Bispebjerg Hospital, Copenhagen, Denmark. \\ e-mail: yong.tang@mssm.edu \\ (Accepted September 24, 2003)
}

\begin{abstract}
Unbiased stereological sampling and counting techniques for estimating the total length, total volume and diameter distribution of myelinated nerve fibers in white matter and the total number of synapses in neocortex of human autopsy brains were described. Uniform random sampling of tissues from entire hemisphere was performed. The total volume and total length of myelinated fibers in white matter were estimated from the product of the volume of white matter obtained with the Cavalieri principle and the volume density and length density of myelinated fibers in white matter, respectively. The volume density of myelinated nerve fibers in white a matter was estimated with a point counting method. The length density of myelinated fibers in white matter was estimated from the isotropic, uniform random sections that were ensured by the sector. The diameter of myelinated fibers was derived by measuring the profile diameter perpendicular to its longest axis. The ethanolic phosphotungstic acid staining technique was modified for staining synapses in human autopsy brains. The total number of synapses in each neocortical region was estimated as the product of the volume of each neocortical region and the numerical density of synapses in each region. The numerical density of synapses in each neocortical region was obtained with the disector at the electron microscopical level. The presented methods will be useful for quantitative studies of the changes of myelinated nerve fibers and synapses in various distinct regions of the central nervous system due to development, aging and diseases.
\end{abstract}

Keywords: human brain, myelinated nerve fibers, neocortex, stereology, synapse, white matter.

\section{INTRODUCTION}

After the first method for estimating the total number of neurons in a distinct region was developed (Sterio, 1984; Gundersen et al., 1986; Gundersen et al., 1988), numerous studies concerning the changes of the neuron number in various regions of brain in normal aging and in a variety of degenerative brain diseases have been performed. These studies have provided many exciting and unexpected results (Morrison and Hof, 1997; Pakkenberg and Gundersen, 1997). The billions of neurons in the central nervous system are connected to one another by means of nerve fibers (dendrites and axons) and synapses to form a complicated network of pathway and neuronal circuits for the transmission of nervous impulses. In order to understand how the brain functions and to explain the causes of brain diseases, it is important to quantitatively investigate the nerve fibers and synapses in the brain. Therefore, the present report extended stereological study of neuron number to stereological study of neuronal connections (myelinated fibers of white matter and synapses of neocortex) in human brain.

\section{MATERIALS}

The materials for the study of myelinated nerve fibers in brain white matter comprised 5 female brains from young autopsy cases, average age 38 years, and 5 female brains from old autopsy cases,

\# "The present paper is a part of the PhD Thesis that Yong Tang prepared during his stay in the Stereological Research Laboratory at the University of Aarhus, Denmark." His current addres is: Neurobiology of Aging Laboratories, Mount Sinai School of Medicine, Box 1639, One Gustave L. Levy Place, New York, NY 10029, USA 
average age 74 years. The human material for the study of neocortical synapses in human brain consisted of five male brains from young autopsy cases (average age 21.8 years) with the post-mortem fixation delay of 2.5 days or less. Excluded were all individuals with prior neurological or psychiatric disorders, or with a medical record of any diseases that may affect the central nervous system and patients with records of alcohol or drug abuse.

\section{METHODS}

\section{STEREOLOGY OF MYELINATED NERVE FIBERS OF WHITE MATTER IN HUMAN BRAIN}

\section{Estimation of the volume of white matter}

The brains were fixed in $0.1 \mathrm{M}$ sodium phosphate buffered formaldehyde for at least 5 months, the meninges removed and the cerebellum and brain stem detached at mid pons. Right or left hemispheres were chosen at random. The hemispheres were embedded in $6 \%$ agar, sliced coronally at an average of $7-\mathrm{mm}$ wide intervals, starting randomly at the frontal pole. A transparent counting grid was placed at random over the occipital cut surface of every brain slice (Fig. 1). The volume of brain white matter was calculated according to the Cavalieri's principle (Gundersen and Jensen 1987; Tang and Nyengaard, 1997; Tang et al., 1998):

$$
V=\overline{\boldsymbol{t}} \times(a / p) \times \Sigma P .
$$

Where $V$ is the total volume of white matter, $\bar{t}=$ $7 \mathrm{~mm}$ is the average slice thickness, $(a / p)$ the area associated with each point in the grid, and $\Sigma P$ the total number of points hitting white matter.

\section{Uniform random sampling of tissue blocks from white matter}

From the brain slices, every fourth slice was sampled systematically, the first one being chosen at random among the first four slices. From the sampled slices, about 20 tissue blocks were uniformly sampled from white matter using a biopsy needle with a diameter of $1.5 \mathrm{~mm}$ and a randomly positioned plastic sheet perforated with equidistantly spaced holes. That is, the tissue blocks were sampled where the holes in the randomly positioned plastic sheet hit white matter. Eight tissue blocks were randomly sampled again.

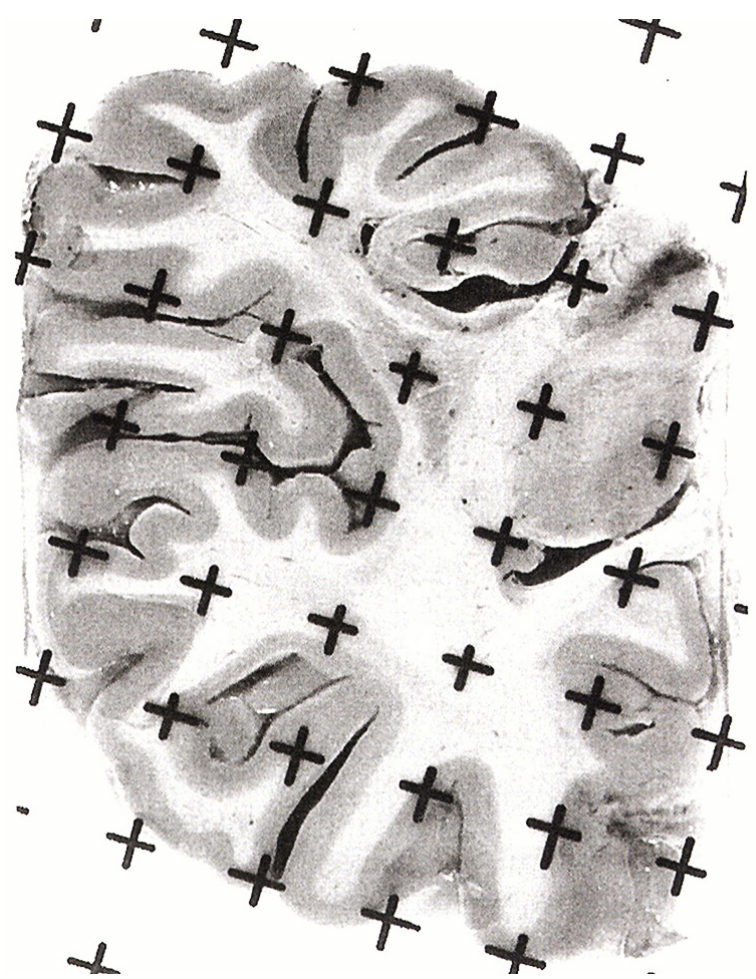

Fig. 1. A coronally cut surface of a human brain with a transparent test system superimposed at random. For the Cavalieri estimates of the total volume of white matter and cortex, all the test points hitting white matter and cortex were counted respectively.

\section{Estimation of the length density and volume density of myelinated nerve fibers in white matter}

The blocks sampled from brain white matter were rinsed for 20 minutes in maleic acid buffer, postfixed for $1 \mathrm{~h}$ in $1 \%$ buffered osmium tetroxide, dehydrated, and then stained with uranyl acetate. Isotropic, uniform random sections were generated by the isector (Nyengaard and Gundersen, 1992) where the block was embedded in a sphere of Epon, which was rolled freely before being re-embedded in Epon for sectioning. One section with thickness of $0.1 \mu \mathrm{m}$ was cut from each Epon block using a LKB Historange microtome. These sections were stained with $1 \%$ toluidine blue. The sections were viewed using a modified Olympus BH-2 microscope. A personal computer and a monitor were connected to a colour video camera mounted on top of the microscope. By means of the CAST-Grid software (Olympus Denmark), the stereological probes (points and counting frames) were superimposed on the video images of the tissue sections viewed on the monitor. The area of each counting frame was $165 \mu \mathrm{m}^{2}$ at the tissue level. An oil objective lens (100X, NA 1.40) 
and an intermediate lens $(5 \mathrm{X})$ before the video camera were used when counting. Using a high light microscopical magnification of 5185X, two fields of vision were randomly sampled in each Epon section, that is, the undamaged tissue of the left upper corner and right lower corner were sampled from the IUR sections of the uniformly sampled blocks.

The length density of myelinated nerve fibers in white matter, $L_{V}$, was estimated as:

$$
L_{V}(n f / w m)=2 \cdot \frac{\Sigma Q(n f)}{\Sigma A(\text { frame })},
$$

where $\Sigma Q(n f)$ denotes the total number of myelinated nerve fiber profiles that were counted in each brain white matter using the unbiased 2-dimensional counting frame (Gundersen, 1977) (Fig. 2), $\Sigma$ A(frame) is the total areas of the unbiased counting frame used when counting the fiber profiles, and 2 is a constant pertaining to isotropic, uniform random sections.

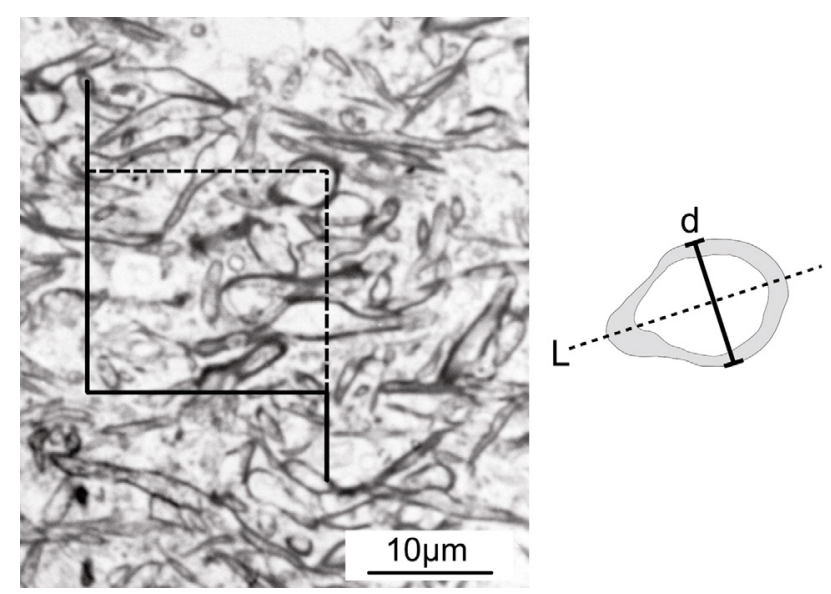

Fig. 2. A uniform random sampling of a field of vision from an IUR section of brain white matter with an unbiased counting frame superimposed is shown to illustrate the way to estimate the length density and diameter of the myelinated fibers in brain white matter. To provide an isotropic, uniform random (IUR) section, the tissue was embedded according to the isector technique. The myelinated fiber profiles were sampled by the use of the two-dimensional unbiased counting frame where the area was indicated by the full drawn lines and the dotted lines (left picture). All myelinated fiber profiles inside the counting frame, provided they did not touch or intersect the full-drawn exclusion lines, were considered for counting. The diameter of myelinated fibers sampled was estimated by measuring the profile diameter perpendicular to their longest axis (right schematic drawing); d: diameter; L: longest axis. Bar is $10 \mu \mathrm{m}$.
The volume density of myelinated nerve fibers in white matter, $\boldsymbol{V}_{\boldsymbol{V}}$, was estimated as:

$$
V_{V}(n f / w m)=\frac{\sum P(n f)}{\sum P(w m)}
$$

where $\Sigma P(n f)$ is the total number of points hitting myelinated nerve fibers within white matter, and $\Sigma P(w m)$ is the total number of points hitting white matter.

\section{Estimation of the total length, total volume and diameter of myelinated nerve fibers in white matter}

To obtain the total length and total volume of myelinated nerve fibers in each brain white matter, the length density and volume density of myelinated fibers in white matter were multiplied by twice of the total white matter volume per hemisphere, $\mathrm{V}(\mathrm{wm})$, i.e., the total white matter volume per brain. Assuming that the myelinated nerve fibers are cylindrical tubes, the diameter of the nerve fibers was derived by measuring the longest profile diameter perpendicular to its longest axis (Tang and Nyengaard, 1997; Tang et al., 1998).

\section{Estimation of processing-induced tissue shrinkage in white matter}

Two tissue slabs, $2 \mathrm{~mm}$ long by $1.5 \mathrm{~mm}$ wide, were taken from the white matter in all hemispheres. The dimensions of these pieces of tissue were measured carefully before being processed. The tissue cross sectional area was computed. The tissue was then dehydrated, embedded, sectioned and stained. After being processed, the area of the tissue sections was measured using point counting:

$$
A=\Sigma P \times(a / p)
$$

where $\Sigma P$ is the total number of points hitting tissue, and $(a / p)$, is the area associated with each point of the counting grid. The measurements were then compared to see if any shrinkage had occurred. The amount of shrinkage was estimated as:

$$
\begin{gathered}
\text { Areal shrinkage }=[(\text { area before })- \\
-(\text { area after })] /(\text { area before })
\end{gathered}
$$




\section{STEREOLOGY OF SYNAPSES OF NEOCORTEX IN HUMAN BRAIN}

\section{Estimation of the volume of neocortex and uniform random sampling of tissue blocks from each neocortical region}

The four neocortical regions, i.e., frontal, temporal, parietal and occipital cortex, were delineated on the pial surface using different colors (Pakkenberg and Gundersen, 1997). The volume of each neocortical region, $V(r e g)$, was estimated with the same way used for the estimation of white matter volume. Every second brain slice was sampled in a systematic random manner. The sampled slices from the same neocortical region (frontal, parietal, temporal and occipital) were identified. Two tissue blocks were uniformly randomly sampled from each region of neocortex.

\section{Tissue preparation for EM and E-PTA staining of synapses}

The sampled tissue blocks were embedded in agar to stabilize them for the subsequent cutting on a vibratome. The blocks were cut into $75 \mu \mathrm{m}$-thickslices using a vibratome (Micro-cut H 1200, Bio-Rad, USA) and the slices were then put into $0.1 \mathrm{M}$ phosphate buffer $(\mathrm{pH}=7.4)$ for one day. The slices were treated with a mixture of osmium and maleic acid for half a hour and rinsed three times in the buffer at room temperature. Subsequently, the slices were dehydrated in a graded series of ethanol solutions (30-50-70-90\%), 4 min each at room temperature. The slices were stained for $2.5 \mathrm{hrs}$ at 60 ${ }^{\circ} \mathrm{C}$ in a $1 \%$ ethanolic phosphotungstic acid (E-PTA) solution containing a trace of water. Then, the slices were treated with water-free acetone (two changes, 10 min each) at $4^{\circ} \mathrm{C}$ and kept in a mixture $(1: 1)$ of acetone and Epon for 1.5 hour at room temperature. In order to embed the slices as flatly as possible a small tube containing hardened Epon was used as an embedding mould and the slice was placed in the mould and covered with a drop of Epon. The embedded blocks were dried in an oven at $60^{\circ} \mathrm{C}$ for at least 24 hours.

\section{Preparation of serial ultrathin sections and micrographs and estimation of section thickness}

The embedded block was cut into sections with a thickness of 50-90 nm using an LKB 2088 ultramicrotome (Sweden). Ribbons of six to seven consecutive sections were picked up on a single hole grid that was covered with a Formvar film. Since the tissue blocks were uniformly random sampled from the neocortex of human autopsy brains, sections could be arbitrarily selected from the ribbons under the condition that the selection of sections is independent of synapses inside sections. The criteria for the selection of a disector pair was that at least one fold was present in each of the two sections. In order to increase efficiency, the thicker sections were preferentially selected. The first two consecutive sections that both had folds were then chosen and examined in a Phillips 300 electron microscope. Since the same positions had to be photographed in corresponding parts of consecutive ultrathin sections, the photographs were taken from undamaged places that were very close to the corners of the sections. Electron micrographs were enlarged to a final magnification of $\sim 12000 \times$. A magnification standard (grating replica) was photographed and printed together with each series of electron micrographs. At least one fold and until three folds appearing on each chosen section were photographed and used for the estimation of section thickness. The thickness of the ultrathin sections was estimated with Small's smallest method (Small, 1968). The folds were photographed at a magnification of $\sim 50,000$. The smallest fold width was measured on the photograph. Since minimal folds stand perpendicular to the section plane and have a width that equals twice that of the section thickness the ultrathin section thickness was half of the smallest fold width obtained.

\section{Estimation of synaptic density in neocortex using the disector principle}

The physical disector principle (Sterio, 1984) was used to count synapses (Tang et al., 2001). The postsynaptic density was used as a counting unit. The electron micrograph from the first section was termed the reference plane and the micrograph from the next ultrathin section was called the look-up plane (Fig. 3). A transparency with an unbiased counting frame (Gundersen, 1977) was superimposed on each of the micrographs. A distance (guard area) that exceeded the largest dimension of the largest postsynaptic density separated the edges of the frame and the micrograph. Synapses were labeled on the reference micrograph if their postsynaptic density profiles were located either entirely or partly within the counting frame and did not intersect the forbidden lines or their extensions. The synapses and "synapse bridges" present in the reference plane and not in the look-up plane were counted and synapses marked in both planes were thus not counted (Fig. 3). 
The numerical density of synapses in each region, $N_{V}($ syn/reg), was estimated as:

$$
N_{V}(\text { syn } / \text { reg })=\frac{\sum Q^{-}(\text {syn })}{\sum v(\text { dis })}=\frac{\sum Q^{-}(\text {syn })}{\sum[A(\text { frame }) \cdot h(\text { dis })]},
$$

where $\Sigma Q^{-}(s y n)$ is the total number of synapses minus the total number of "synapse bridges" in the disectors per region of neocortex and $\Sigma v$ (dis) is the total volume of the disectors. The volume of a given disector is calculated by multiplying the area of the counting frame, a (frame), by the height of the disector, $h$ (dis), which in this case is the sum of the two section thicknesses, because $\Sigma Q^{-}($syn) was obtained by counting both ways.

\section{Estimation of the total number of synapses}

The total number of synapses in each neocortical region, $N($ syn,reg), was calculated as:

$$
N(\text { syn, reg })=N_{V}^{(s y n / r e g)} \cdot V(r e g) .
$$

The total number of synapses in the neocortex of a brain is the sum of the number of synapses in the four regions. The total number of neurons in neocortex was estimated in another study (Pakkenberg and Gundersen, 1997). The number of synapses per neuron, $N_{N}(s y n / n e u)$, was obtained by dividing the total number of synapses by the total number of neurons in neocortex.

\section{Estimation of processing-induced tissue shrinkage in neocortex}

All eight tissue blocks sampled per brain were used for the estimation of shrinkage. Before being processed, the cross sectional areas of the tissue blocks were measured using point counting. The tissue blocks were then processed and embedded in Epon as described previously. After Epon embedding, the areas of the tissue blocks were measured again using point counting. The areal shrinkage was estimated with the same way used in white matter. The processing-induced volume shrinkage for one brain was estimated as:

Volume shrinkage $=$ mean $\left[1-\left[\frac{A(\text { after })}{A \text { (before })}\right]^{3 / 2}\right]$,

where $A$ (before) is the area of a tissue block before being processed, $A$ (after) is the area of the same tissue block in the Epon, and mean is the simple arithmetic mean over the eight blocks.

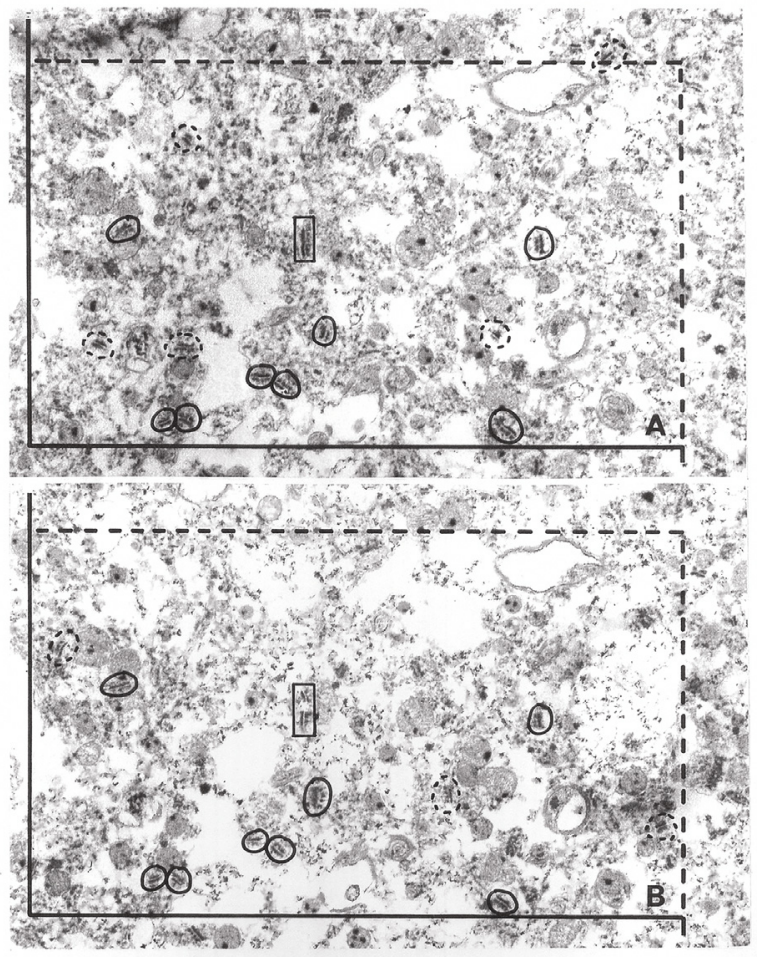

Fig. 3. Two consecutive ultrathin sections from the neocortex of a human brain are shown to illustrate the disector method for counting synapses. Unbiased sampling frames are superimposed on the pair of micrographs. The synapses present (dashed circle) in the reference plane and not in the look-up plane were counted if their postsynaptic densities were completely or partly inside the sampling frame without touching the exclusion lines and their extension (solid lines). In the look-up plane, the counting frame is not considered at all. The unbiased counting frame is illustrated on both planes in this illustration to indicate that synapses were counted both ways: first $A$ was considered the look-up plane allowing one to count synapses in the frame in $B$, then the role of the two sections was reversed. The two separate synapse profiles marked with full square in Fig. B were found to belong to one complex synapse when examining the consecutive section shown in Fig. $A$. This event is called synapse bridge. When calculating the numerical density of synapses the number of synapse bridge is subtracted from the number of synapses. The length of the horizontal line of the counting frame corresponds to $13 \mu \mathrm{m}$.

\section{STATISTICS}

Group means were compared using Student's ttest for unpaired observations. A $2 p$ value of 0.05 was adopted throughout. Variability within groups was estimated using the dimensionless coefficient of variation $(\mathrm{CV}=\mathrm{SD} / \mathrm{Mean})$. 
The precision of estimates is expressed as the coefficient of error, $C E=S E M /$ Mean . Depending on the estimator and the sampling strategy, $C E$ is estimated according to a quite wide ranges of equations described below, all of which are just robust approximations. No exact estimators of variability exist for the estimators and sampling strategies used in this report.

The total length and total volume of myelinated fibres in white matter and the total number of synapses in neocortex were calculated from the product of density estimates and white matter volume and neocortical volume. Therefore, the total inter-brain $\mathrm{CE}$ has to be calculated from these two-step analyses.

$\mathrm{CE}$ for the Cavalieri estimate of the white matter volume, $C E[V(\mathrm{wm})]$, and for the Cavalieri estimate of the volume of neocortex, $C E[V(n e o)]$, was computed from the expected variance of point counting with a lattice of systematic points on white matter and cortex of brain, respectively, Var $_{\text {Noise }}$ (white matter) and $\operatorname{Var}_{\text {Noise }}$ (cortex), and the variance of areas among the systematic random sample of slices used to estimate the volume of white matter and cortex, $\operatorname{Var}_{S R S}[a(w m)]$ and $\operatorname{Var}_{S R S}[a(\operatorname{cortex})]$, respectively (Gundersen et al., 1999).

Var $_{\text {Noise }}$ was computed as:

$$
\operatorname{Var}_{\text {Noise }}=0.0724 \cdot(b / \sqrt{a}) \cdot \sqrt{n_{b} \cdot \sum_{i=1}^{n} P_{i}} \text {, }
$$

where 0.0724 is a constant for point lattices with a quadratic arrangement, $\boldsymbol{b} / \sqrt{\boldsymbol{a}}$ is an average shape factor for a set of sectional profiles, i.e., the length of the profile boundary, $b$, divided by the square root of the profile area, $\sqrt{\boldsymbol{a}}, n_{b}$ is the number of slices used, and $P_{i}$ is the number of points hitting white matter and cortex, respectively, on the $i$ th slice.

The CE for the estimates of the volume of white matter and cortex was estimated as:

$$
\begin{aligned}
& C E(V)=\frac{\sqrt{\text { Var Noise }+ \text { Var SRS }}}{\sum_{i=1}^{n} P_{i}}= \\
& \frac{\sqrt{\text { Var Noise }+[3(A-\text { Var Noise })-4 B+C] / 240}}{\sum_{i=1}^{n} P_{i}} \\
& .
\end{aligned}
$$

Where $A=\sum\left(\mathrm{P}_{\mathrm{i}} \cdot \mathrm{P}_{\mathrm{i}}\right), B=\sum\left(\mathrm{P}_{\mathrm{i}} \cdot \mathrm{P}_{\mathrm{i}+1}\right)$, and $C=\sum$ $\left(\mathrm{P}_{\mathrm{i}} \cdot \mathrm{P}_{\mathrm{i}+2}\right)$.
Since the estimators of the length density and volume density of myelinated fibers in white matter are ratio estimators, the one-pass equation in Kroustrup and Gundersen (1983) was used to estimate their CE.

$C E\left[L_{V}(n f / w m)\right]$ and $C E\left[V_{V}(n f / w m)\right]$ were calculated according to the following formula:

$$
\begin{aligned}
& C E_{n}\left(\frac{\sum Y}{\sum X}\right)= \\
& \qquad \sqrt{\frac{n}{n-1}\left(\frac{\sum(X)^{2}}{\sum X \sum X}+\frac{\sum(Y)^{2}}{\sum Y \sum Y}-\frac{2 \sum(X Y)}{\sum X \sum Y}\right)}
\end{aligned}
$$

Where $\Sigma X$ is the number of fields of vision used in each block when estimating the length density of myelinated fibers in white matter or number of the fields of vision used in each block when estimating the volume density of myelinated fibers in white matter. $\Sigma Y$ is the number of the fiber profiles in each block or number of the points hitting the fiber profiles in each block and $n$ is the number of blocks used when estimating the length density or volume density of myelinated fibers in white matter.

The CE for the estimates of the total length and total volume of myelinated fibers in the white matter per brain was calculated using the formulae:

$$
\begin{aligned}
& C E[L(n f, w m)]= \\
& \sqrt{C E^{2}[L V(n f / w m)]+C E^{2}[V(w m)]}, \\
& C E[V(n f, w m)]= \\
& \sqrt{C E^{2}[V V(n f / w m)]+C E^{2}[V(w m)]} .
\end{aligned}
$$

The CE for the estimation of synapse density in neocortex, $C E\left[N_{V}(s y n / n e o)\right.$, was calculated according to the following formula:

$$
\begin{aligned}
& C E\left[N_{V}(\text { syn/neo })\right]= \\
& \sqrt{\frac{n}{n-1}\left[\frac{\sum\left(Q^{-}\right)^{2}}{\left(\sum Q^{-}\right)^{2}}+\frac{\sum(v(\text { dis }))^{2}}{\left(\sum v(\text { dis })\right)^{2}}-\frac{2 \sum\left(Q^{-} \cdot v(\text { dis })\right)}{\sum Q^{-} \cdot \sum v(\text { dis })}\right]}
\end{aligned}
$$

where $n$ is the number of disector positions in all blocks, $\Sigma \boldsymbol{Q}^{-}$is the total number of synapses counted in all disectors, and $\sum v($ dis $)$ is the total volume of disectors used.

The CE for the estimate of the total number of synapses in the neocortex per brain was calculated using the formula: 


$$
\begin{aligned}
& C E[N(\text { syn, neo })]= \\
& \sqrt{C E^{2}[V(\text { neo })]+C E^{2}\left[N_{V}(\text { syn } / \text { neo })\right]} \cdot
\end{aligned}
$$

\section{RESULTS}

The stereological results of white matter and myelinated fibers in white matter from 10 female human brains are presented in Table 1 . The mean processing-induced shrinkage of the brain white matter of young and old subjects was $1.5 \%$ and $6.7 \%$, respectively; none of them differed significantly from zero. The total length of myelinated fibers in the white matter in the old group was significantly reduced by $27 \%$ compared with the young group ( $2 p$ $=0.01)$ (Table 1). On average, 300 myelinated fiber profiles were counted per brain with a mean $\mathrm{CE}$ of
0.07. On average, 300 points hitting on the myelinated fiber profiles were counted per brain with a mean $\mathrm{CE}$ of 0.06 .

The stereological results of synapses from five human brain neocortices are presented in Table 2 . The mean volume shrinkage of the human neocortex induced by the histological processing was $22 \%$. The numerical density of synapses in neocortex was corrected for the processing-induced shrinkage of neocortex. The total number of synapses in the entire neocortex was $16510^{12}(0.16)$ with an overall synaptic density of $\sim 28010^{6} / \mathrm{mm}^{3}$. The number of synapses per neuron was $6.94 \cdot 10^{3}(0.16)$. On average, 32 disectors were used and 288 synapses were counted per hemisphere with a mean CE of 0.14 (Table 2) for the estimate of the total number of synapses in neocortex.

\begin{tabular}{|c|c|c|c|c|c|c|c|c|}
\hline $\begin{array}{c}\text { Age } \\
\text { (Years) }\end{array}$ & $\begin{array}{c}\mathrm{V}(\mathrm{wm}) \\
\left(\mathrm{cm}^{3}\right)\end{array}$ & $\begin{array}{l}\mathrm{CE} \\
(\%)\end{array}$ & $\mathrm{V}_{\mathrm{V}}(\mathrm{NF})$ & $\begin{array}{l}\mathrm{CE} \\
(\%)\end{array}$ & $\begin{array}{l}\mathrm{V}(\mathrm{NF}) \\
\left(\mathrm{cm}^{3}\right)\end{array}$ & $\begin{array}{l}\mathrm{CE} \\
(\%)\end{array}$ & $\begin{array}{r}\mathrm{L}_{\mathrm{V}}(\mathrm{NF}) \\
\left(\mathrm{km} / \mathrm{cm}^{3}\right)\end{array}$ & $\begin{array}{l}\mathrm{CE} \\
(\%)\end{array}$ \\
\hline \multicolumn{9}{|c|}{ Young subjects } \\
\hline 18 & 494 & 2.4 & 0.35 & 5.6 & 173 & 6.1 & 244 & 6.3 \\
\hline 30 & 464 & 2.3 & 0.33 & 3.1 & 153 & 3.9 & 245 & 4.7 \\
\hline 39 & 488 & 5.1 & 0.32 & 2.9 & 156 & 5.9 & 249 & 4.8 \\
\hline 45 & 398 & 2.6 & 0.34 & 2.5 & 135 & 3.6 & 264 & 6.7 \\
\hline 57 & 530 & 2.0 & 0.31 & 3.6 & 164 & 4.1 & 244 & 5.6 \\
\hline Mean & $475(10)^{*}$ & 3.1 & $0.33(4.8)$ & 3.7 & $156(9.1)$ & 4.8 & 249 (3.4) & 5.7 \\
\hline \multicolumn{9}{|c|}{ Old subjects } \\
\hline 62 & 473 & 3.5 & 0.29 & 6.3 & 137 & 7.2 & 234 & 6.6 \\
\hline 68 & 410 & 5.2 & 0.27 & 5.4 & 111 & 7.5 & 195 & 6.1 \\
\hline 70 & 290 & 2.3 & 0.30 & 4.3 & 87 & 4.9 & 209 & 6.4 \\
\hline 80 & 378 & 4.1 & 0.38 & 4.1 & 144 & 5.8 & 201 & 4.5 \\
\hline 90 & 462 & 5.0 & 0.36 & 5.4 & 166 & 7.4 & 220 & 6.3 \\
\hline Mean & $403(18)$ & 4.0 & $0.32(15)$ & 5.1 & $129(24)$ & 6.6 & $212(7.3)$ & 6.0 \\
\hline $\begin{array}{c}\text { Age } \\
\text { (Years) }\end{array}$ & $\begin{array}{l}\mathrm{L}(\mathrm{NF}) \\
10^{3} \mathrm{Km}\end{array}$ & $\begin{array}{l}\text { CE } \\
(\%) \\
\end{array}$ & $\begin{array}{c}\mathrm{D}(\mathrm{NF}) \\
(\mu \mathrm{m})\end{array}$ & $\begin{array}{l}\mathrm{CE} \\
(\%) \\
\end{array}$ & $\begin{array}{c}\text { CV (D) } \\
(\%)\end{array}$ & & & \\
\hline \multicolumn{9}{|c|}{ Young subjects } \\
\hline 18 & 121 & 6.7 & 1.19 & 2.7 & 49.2 & & & \\
\hline 30 & 114 & 5.2 & 1.21 & 2.7 & 48.9 & & & \\
\hline 39 & 122 & 7.0 & 1.10 & 2.8 & 50.7 & & & \\
\hline 45 & 105 & 7.2 & 1.09 & 2.8 & 55.4 & & & \\
\hline 57 & 129 & 5.9 & 1.12 & 3.1 & 53.8 & & & \\
\hline Mean & $118(7.7)$ & 6.4 & $1.14(4.8)$ & 2.8 & 51.6 & & & \\
\hline \multicolumn{9}{|c|}{ Old subjects } \\
\hline 62 & 111 & 7.5 & 1.23 & 2.8 & 48.8 & & & \\
\hline 68 & 80 & 8.0 & 1.25 & 3.2 & 52.9 & & & \\
\hline 70 & 61 & 6.8 & 1.14 & 2.9 & 50.5 & & & \\
\hline 80 & 76 & 6.1 & 1.39 & 3.2 & 53.5 & & & \\
\hline 90 & 102 & 8.0 & 1.37 & 3.3 & 55.3 & & & \\
\hline Mean & $86(23)$ & 7.3 & $1.28(8.1)$ & 3.1 & 52.2 & & & \\
\hline
\end{tabular}

Table 1. The stereological results of human brain white matter and the myelinated nerve fibers therein.

$\mathrm{V}(\mathrm{wm})$ : mean volume of white matter; $\mathrm{V}_{\mathrm{V}}(\mathrm{NF})$ : volume density of nerve fibers; $\mathrm{V}(\mathrm{NF})$ : total volume of nerve fibers; $\mathrm{L}_{\mathrm{V}}(\mathrm{NF})$ : length density of nerve fibers; L (NF): total length of nerve fibers; D (NF): geometric mean diameter of nerve fibers; CE: coefficient of error; CV (D): coefficient of variation of nerve fiber diameter distribution. * The numbers in parentheses are the observed inter-individual coefficients of variation in percent. 
Table 2. The results of synapses from all five human brains.

\begin{tabular}{|c|c|c|c|c|c|c|c|}
\hline No. / Region & $\begin{array}{l}\mathrm{V}(\text { neo }) \\
\mathrm{Cm}^{3}\end{array}$ & $\begin{array}{l}\mathrm{CE} \\
(\%)\end{array}$ & $\sum \mathrm{Q}^{-}(\mathrm{syn})$ & $\sum \mathrm{Q}($ syn $)$ & $\begin{array}{l}\mathrm{h}(\text { dis }) \\
\mathrm{nm}\end{array}$ & & \\
\hline \multicolumn{8}{|l|}{$\overline{\text { Brain No. } 1}$} \\
\hline Occipital & 67 & 5.6 & 58 & & & & \\
\hline Parietal & 155 & 4.2 & 86 & & & & \\
\hline Temporal & 152 & 5.3 & 117 & & & & \\
\hline Frontal & 247 & 7.9 & 97 & & & & \\
\hline Total / Average & 621 & 3.7 & 358 & 649 & 65 & & \\
\hline \multicolumn{8}{|l|}{ Brain No. 2} \\
\hline Occipital & 57 & 5.2 & 72 & & & & \\
\hline Parietal & 151 & 2.8 & 75 & & & & \\
\hline Temporal & 129 & 2.5 & 70 & & & & \\
\hline Frontal & 195 & 5.2 & 59 & & & & \\
\hline Total / Average & 532 & 2.4 & 276 & 484 & 55 & & \\
\hline \multicolumn{8}{|l|}{ Brain No. 3} \\
\hline Occipital & 71 & 4.9 & 62 & & & & \\
\hline Parietal & 152 & 4.9 & 96 & & & & \\
\hline Temporal & 156 & 3.0 & 88 & & & & \\
\hline Frontal & 278 & 4.5 & 82 & & & & \\
\hline Total / Average & 657 & 2.1 & 328 & 626 & 77 & & \\
\hline \multicolumn{8}{|l|}{ Brain No. 4} \\
\hline Occipital & 60 & 4.9 & 62 & & & & \\
\hline Parietal & 135 & 1.7 & 95 & & & & \\
\hline Temporal & 117 & 6.6 & 53 & & & & \\
\hline Frontal & 233 & 5.4 & 58 & & & & \\
\hline Total / Average & 545 & 2.9 & 268 & 484 & 58 & & \\
\hline \multicolumn{8}{|l|}{ Brain No. 5} \\
\hline Occipital & 90 & 4.1 & 68 & & & & \\
\hline Parietal & 151 & 5.5 & 65 & & & & \\
\hline Temporal & 110 & 4.4 & 41 & & & & \\
\hline Frontal & 243 & 3.1 & 36 & & & & \\
\hline Total / Average & 594 & 2.0 & 210 & 380 & 61 & & \\
\hline \multicolumn{8}{|l|}{ Mean results } \\
\hline Occipital & 69 (19) & 4.9 & 64 & & & & \\
\hline Parietal & $149(5)$ & 4.1 & 83 & & & & \\
\hline Temporal & $133(16)$ & 4.6 & 73 & & & & \\
\hline Frontal & $239(12)$ & 5.4 & 66 & & & & \\
\hline Total/Average & $590(9)$ & 2.7 & 288 & 525 & 63 & & \\
\hline No. / Region & $\begin{array}{l}\mathrm{CE}(\mathrm{t}) \\
(\%)\end{array}$ & $\begin{array}{l}\overline{\mathrm{h}}(\text { syn) } \\
\mathrm{nm}\end{array}$ & $\begin{array}{l}\mathrm{N}_{\mathrm{V}}(\text { syn }) \\
\mu \mathrm{m}^{-3}\end{array}$ & $\begin{array}{l}\mathrm{CE} \\
(\%)\end{array}$ & $\begin{array}{l}\text { N(syn) } \\
10^{12} \\
\end{array}$ & $\begin{array}{l}\mathrm{CE} \\
(\%)\end{array}$ & $\begin{array}{l}\mathrm{N}(\text { syn/neu }) \\
10^{3}\end{array}$ \\
\hline \multicolumn{8}{|l|}{ Brain No. 1} \\
\hline Occipital & & 104 & 0.21 & & 14.0 & & 3.28 \\
\hline Parietal & & 99 & 0.31 & & 47.8 & & 6.87 \\
\hline Temporal & & 129 & 0.36 & & 55.3 & & 8.42 \\
\hline Frontal & & 139 & 0.27 & & 65.7 & & 8.75 \\
\hline Total / Average & 1.8 & 123 & 0.29 & 10 & 183 & 14 & 7.22 \\
\hline \multicolumn{8}{|l|}{ Brain No. 2} \\
\hline Occipital & & 107 & 0.27 & & 15.3 & & 2.94 \\
\hline Parietal & & 103 & 0.27 & & 40.2 & & 6.02 \\
\hline Temporal & & 108 & 0.27 & & 34.7 & & 8.29 \\
\hline Frontal & & 103 & 0.21 & & 40.1 & & 5.08 \\
\hline Total / Average & 1.0 & 105 & 0.24 & 15 & 130 & 16 & 5.44 \\
\hline \multicolumn{8}{|l|}{ Brain No. 3} \\
\hline Occipital & & 180 & 0.16 & & 11.0 & & 3.34 \\
\hline Parietal & & 140 & 0.29 & & 43.4 & & 8.67 \\
\hline
\end{tabular}




\begin{tabular}{|c|c|c|c|c|c|c|c|}
\hline No. / Region & $\begin{array}{l}\mathrm{CE}(\mathrm{t}) \\
(\%)\end{array}$ & $\begin{array}{l}\bar{h} \text { (syn) } \\
\mathrm{nm}\end{array}$ & $\begin{array}{l}N_{V} \text { (syn) } \\
\mu m^{-3}\end{array}$ & $\begin{array}{l}\mathrm{CE} \\
(\%)\end{array}$ & $\begin{array}{l}\mathrm{N}(\text { syn }) \\
10^{12}\end{array}$ & $\begin{array}{l}\mathrm{CE} \\
(\%)\end{array}$ & $\begin{array}{l}\mathrm{N}(\mathrm{syn} / \mathrm{neu}) \\
10^{3}\end{array}$ \\
\hline Temporal & & 129 & 0.27 & & 42.4 & & 9.81 \\
\hline Frontal & & 118 & 0.27 & & 75.2 & & 10.1 \\
\hline Total / Average & 3.4 & 133 & 0.26 & 10 & 172 & 11 & 8.58 \\
\hline \multicolumn{8}{|l|}{ Brain No. 4} \\
\hline Occipital & & 104 & 0.25 & & 15.2 & & 4.92 \\
\hline Parietal & & 99 & 0.38 & & 51.7 & & 9.02 \\
\hline Temporal & & 126 & 0.16 & & 18.4 & & 4.94 \\
\hline Frontal & & 103 & 0.26 & & 59.7 & & 7.23 \\
\hline Total / Average & 5.4 & 107 & 0.27 & 16 & 145 & 16 & 6.97 \\
\hline \multicolumn{8}{|l|}{ Brain No. 5} \\
\hline Occipital & & 122 & 0.61 & & 54.9 & & 7.45 \\
\hline Parietal & & 108 & 0.36 & & 54.0 & & 6.25 \\
\hline Temporal & & 120 & 0.29 & & 31.9 & & 6.13 \\
\hline Frontal & & 117 & 0.21 & & 51.8 & & 6.18 \\
\hline Total / Average & 2.8 & 116 & 0.32 & 15 & 193 & 15 & 6.51 \\
\hline \multicolumn{8}{|l|}{ Mean results } \\
\hline Occipital & & 123 & $0.30(60)$ & & $22.1(83)$ & & $4.38(43)$ \\
\hline Parietal & & 110 & $0.32(15)$ & & $47.4(12)$ & & $7.36(19)$ \\
\hline Temporal & & 122 & $0.27(27)$ & & $36.5(37)$ & & $7.52(26)$ \\
\hline Frontal & & 116 & $0.24(13)$ & & $58.5(23)$ & & $7.48(27)$ \\
\hline Total/Average & 3.2 & 117 & $0.28(11)$ & 14 & $165(16)$ & 14 & $6.94(16)$ \\
\hline
\end{tabular}

$\mathrm{V}\left(\right.$ neo): total volume of neocortex; $\sum \mathrm{Q}^{-}(\mathrm{syn})$ : total number of synapses counted in disectors; $\Sigma \mathrm{Q}$ (syn): total synaptic profiles counted in reference and sampling sections; $\mathrm{h}$ (dis): average height of dissectors; $\mathrm{CE}(\mathrm{t})$ : error in estimate of sections thickness; $\overline{\mathrm{h}}(\mathrm{syn})$ : mean projected height of synapses; $\mathrm{N}_{\mathrm{V}}(\mathrm{syn})$ : numerical density of synapses in neocortex; $\mathrm{N}(\mathrm{syn})$ : total number of synapses; $\mathrm{N}(\mathrm{syn} / \mathrm{neu})$ : number of synapses per neuron. The coefficient of error of individual estimates, CE, is shown next to the quantity it refers to. The coefficient of variation in percentage is shown in brackets for various estimates. The numerical density of synapses, the total number of synapses and number of synapses per neuron were corrected for the tissue shrinkage induced by tissue processing.

\section{DISCUSSION}

\section{UNBIASED ESTIMATES BASED ON A UNIFORM RANDOM SAMPLING DESIGN}

The sampling strategy of earlier studies on nerve fibers of white matter and synapses of neocortex was to sample a certain typical portion of a brain region of interest. The conclusions that can be drawn from the analysis of a small part of tissue, a standard section or sections in which the objects of interest are best identified, can only apply to this particular part of tissue and these sections. The "ideal tissue" and "ideal field" selections are, by definition, not representative of the entire tissue and, thus, introduce a bias due to non-uniform sampling. When proper (systematic) uniform random sampling is carried out, all parts of a region under consideration have an equal probability of being sampled. Then, the mean of estimates approaches the true value with proper replication. Therefore, the resulting estimate can be defined as an unbiased estimate. Without uniform random sampling, it is essentially impossible to obtain an unbiased estimate. In the present study, brain slices were sampled from hemisphere in a systematic random manner, and from the sampled slices, tissue blocks were uniformly randomly sampled again. Therefore, all fibers in the white matter and all synapses in the neocortex of a complete hemisphere had the same probability of being sampled.

\section{THE IMPORTANCE OF ESTIMATING TOTAL QUANTITIES}

A problematic aspect of almost all previous studies is that only myelinated fibre density in various parts of brain white matter and synaptic density in various parts of brain were estimated, and nothing was known about the volume of white matter and neocortex, and consequently, about the total quantities of the myelinated fibers in the distinct region of white matter and the synapses in the distinct region of neocortex. The density estimates only serve as an intermediate observation that allows the estimation of the total quantities. When reporting the density estimates only, the possible age-related and disease-related changes in the reference space, i.e., the total volume of neuropil, were not taken into account. Shrinkage of the reference volume could 
happen during tissue processing, and this shrinkage may be unequal between young and aged individuals, as well as between control subjects and diseased individuals. In addition, modifications of preparative procedures could differentially alter the reference space dimensions. Therefore, biological conclusions based on density measurements are very ambiguous because it will never be known whether any changes in the density are due to an alteration of the total quantity and/or a change in the reference volume. For example, in Table 1, the volume density of myelinated fibers in brain white matter from the two groups was nearly identical $(0.32$ vs. 0.33$)$, but the total volume of myelinated fibers in the brain white matter from the old group was substantially lower than that from the young group $\left(129 \mathrm{~cm}^{3}\right.$ vs. $\left.156 \mathrm{~cm}^{3}\right)$. The volume density of myelinated fibers in white matter did not contain any information of the total volume of brain white matter. It is likely that the widespread use of density estimates has contributed to the apparent contradictions in the earlier literature.

One of the most important features of the present methods is that they were designed to obtain the total quantities. The total length and total volume of myelinated fibers in the brain white matter and the total number of synapses in the brain neocortex were obtained by multiplying the density estimates with the volume of white matter and the volume of neocortex. The volumes of white matter and neocortex were estimated with the Cavalieri principle. This methodology eliminates the questionable assumption implicit in comparisons of density data, i.e., that the volumes of the regions compared are the same. Therefore, the results can be interpreted unambiguously.

\section{Estimation of the length density of myelinated fibres in brain white matter based on isotropic, uniform random sections}

In the present report, three-dimensional uniform sampling strategy was used to provide unbiased estimation of myelinated fiber length in brain white matter. When very long tubules such as myelinated fibers in brain white matter are transected by a plane, the profile count will be a function of the length of the tubules. However, it is intuitively comprehensible that the number of times that a tubule is cut by a sectioning plane is not only directly proportional to the tubular length but also to the direction of the sectioning plane or tortuosity of the tubule. Therefore, when estimating the length of fibers based on two-dimensional sections, it is required that the fiber profiles should be counted on isotropic, uniform random (IUR) planes unless the nerve fibers are isotropic themselves. In earlier studies, directions of fibers in brain white matter were not taken into account. In the present study, the length of myelinated fibers in brain white matter was estimated from IUR sections generated by the isector (Nyengaard and Gundersen, 1992). The use of IUR sections ensures that every direction of the section plane in space has an equal probability of being sampled and that any bias caused by a possible preferential orientation or distribution of nerve fibres is avoided, so that average estimates unlimitedly approach the true quantity.

\section{Estimation of synaptic density with the disector}

In the past, numerous attempts have been made to estimate the number of synapses in various regions of the central nervous system. However, the previous studies either estimated the two-dimensional density, i.e., number of synaptic profiles per unit area of section, based on a random section, or calculated three-dimensional numerical density by means of model-based stereological methods. There are several inherent problems related to the estimation of twodimensional synaptic profile density. Sampling synaptic profile density fails to take the effects of the size, shape, and orientation of the synapses into account. For example, larger synapses have a higher probability of being counted on a random section than small ones and therefore greater effort in counting will only produce a very precise overestimation. If a postsynaptic density curves, a synapse may be multiply intersected by a single plane of section. These multiple intersections of a synapse may apparently appear as unrelated profiles in a single, thin section. On the other hand, it has been found that small or tangentially cut synaptic profiles, which are produced by sectioning of more than $20 \%$ of all synapses, cannot be reliably recognized in a random section due to their negligible size (Geinisman et al., 1986a). This problem is even more aggravated by the presence of the so-called complex perforated synaptic contacts (Geinisman et al., 1986b). In a random section, a perforated synapse can mistakenly be counted as two or more non-perforated ones or not counted at all if the section passes through the interval between separate postsynaptic density segments. In addition, the synaptic profile density ignores the effects of section thickness on the density estimate. The common assumption of previous studies is that random sections, from which the synaptic profile density is obtained, are true planes without thickness. Obviously, this is not true; even the thickness of an 
extremely thin section can still be quite large in comparison with the size of synapses. The effect of non-zero section thickness on the estimation is called overprojection or Holmes effect. Finally, the synaptic profiles obtained from random sections are also biased by an actual or apparent disappearance of small synaptic profiles from sections due to lost caps or lack of resolution (truncation effects) (Gundersen, 1986). For all the above reasons, the number of synaptic profiles seen in one section has no known relationship to the number of synapses in a threedimensional reference space of volume. Therefore, some investigators calculated the three-dimensional density of synapses using model-based stereological methods. The principles underlying model-based stereological methods have relied on several unverified and unverifiable assumptions regarding the size, shape, and orientation distribution of the particles studied. In general, the synapses have usually been treated by applying a "best fitting" geometric form that was generally accepted to be a convex, flat, circular disc. The difficulty is that the assumptions are approximate and unrealistic. An increasing amount of data has been reported in the literature indicating that synapses have much more complex shapes than a simple disc. Synapses may even exhibit a curvature. The conclusions will be biased to the extent that approximation diverge from reality, and the effects of these potential biases on the results of synapses are impossible to evaluate from the data obtained.

Since a number is a zero-dimensional structural characteristic, the only way it can be sampled and estimated unbiasedly is in three dimensions, i.e., using a volume sampling probe. A simple and unbiased three-dimensional probe is the disector (Sterio, 1984). The central idea of the disector is that arbitrarily shaped particles may be counted unambiguously by using at least two consecutive section planes. The probability that a synapse is hit by the sampling section plane but not by the parallel look-up section plane is the same for both large and small synapses. Therefore, the disector method sampled synapses with a uniform probability in a three-dimensional space. The orientation of the section planes can be completely arbitrarily chosen and the estimation is independent of truncation and overprojection in addition to the fact that it does not require assumption about the size, shape and orientation of synapses. By using, alternatively, both sections of the disector, the efficiency of the estimate is doubled since different synapses are being sampled.

\section{Tissue shrinkage}

In order to obtain estimates of the total length and total volume of myelinated fibers in the white matter and the total number of synapses in the brain neocortex, the density estimates have to be multiplied by the volume of the reference space, i.e., the total volume of white matter and total volume of neocortex. In order to verify the assumption that the reference volume is identical for both estimates, any change in tissue volume, between the tissue used for the Cavalieri estimation of volume and the density estimation, should be estimated. In the study of the myelinated fibers in human brain white matter, we embedded the tissue in Epon. The mean areal shrinkage induced by the histological processing, measured in the $\mathrm{x}$, y-direction, was $1.5 \%$ in the brains of young subjects and $6.7 \%$ in the brains of old subjects respectively, none of which significantly differed from zero. Therefore, the estimates of the total length and size of myelinated fibers in the brain white matter were not corrected for tissue shrinkage. In the study of synapses in human brain neocortex, we stained synapses using the E-PTA method and embedded the tissue in Epon. We found that the mean volume shrinkage induced by the histological processing was $22 \%$. This change is not negligible. The numerical density of synapses was corrected for the volume shrinkage of the reference space. In previous studies, the shrinkage of the reference space due to tissue processing for electron microscope was not taken into account. These can partly explain the conflicting results of previous quantitative studies on synapse changes during aging and various experimental conditions.

\section{CONCLUSIONS}

This study presents for all practical purposes unbiased stereological techniques for estimating the total length, total volume and mean diameters of myelinated nerve fibers in white matter and the total number of synapses in neocortex of human autopsy brain. In order to obtain unbiased estimates of myelinated fibers in white matter and synapses in neocortex, uniform random sampling of entire white matter and neocortex must be performed. When estimating the length of myelinated fibers in white matter, isotropic uniform random sections from the sampled blocks have to be used. When estimating the number of synapses in neocortex, synapses must be 
counted with the disector in a known volume of tissue without making any assumptions about size, shape, and orientation of the synapses in neocortex. The results of myelinated fibers in white matter and synapses in neocortex must be expressed as total quantities in white matter and neocortex so that they can be interpreted unambiguously.

\section{ACKNOWLEDGEMENTS}

The study was supported by the Aarhus University Research Foundation, Leo Nelsen's Foundation, Lundbeck Foundation, Geert Jørgensen's Research Bursary, the Foundation for Advancement of Medical Research and Foundation for Research in Psychiatry, the Foundation for Research in Neurology, the Danish Medical Research Council, and the Beckett Foundation. The superb technical assistance of $\mathrm{H}$. Andersen, A. M. Funder, A. Larsen, and M. Lundorf is highly appreciated.

"The preliminary form of this paper was originally presented at the $\mathrm{XI}^{\text {th }}$ International Congress for Stereology-Beijing Conference, Beijing, China, 4-8 November 2003."

\section{REFERENCES}

Geinisman Y, de Toledo Morrell L, Morrell F (1986a). Loss of perforated synapses in the dentate gyrus: morphological substrate of memory deficit in aged rats. Proc Natl Acad Sci USA 83:3027-31.

Geinisman Y, de Toledo Morrell L, Morrell F (1986b). Aged rats need a preserved complement of perforated axospinous synapses per hippocampal neuron to maintain good spatial memory. Brain Res 398:266-75.

Gundersen HJG (1977). Notes on the estimation of the numerical density at arbitrary profiles: The edge effect. J Microsc 111:219-23

Gundersen HJG (1986). Stereology of arbitrary particles. A review of unbiased number and size estimator and presentation of some new ones, in memory of William
R. Thompson. J Microsc 143:3-45.

Gundersen HJG, Jensen EB (1987). The efficiency of systematic sampling in stereology and its prediction. $\mathrm{J}$ Microsc 147:229-63.

Gundersen HJG, Bagger P, Bendtsen TF, Evans SM, Korbo L, Marcussen N, et al. (1988). The new stereological tools: disector, fractionator, nucleator and point sampled intercepts and their use in pathological research and diagnosis, APMIS 96:857-81.

Gundersen HJG, Jensen EB, Kieu K, Nielsen J (1999). The efficiency of systematic sampling in stereologyreconsidered. J Microsc 193:99-211.

Kroustrup JP, Gundersen HJG (1983). Sampling problems in a heterogeneous organ: quantitation of relative and total volume of pancreatic islets by light microscopy. J Microsc 132:43-55.

Morrison JH, Hof PR (1997). Life and death of neurons in the aging brain. Science 278: 412-9.

Nyengaard JR, Gundersen HJG (1992). The isector: a simple and direct method for generating isotropic, uniform random sections from small specimens. J Microsc 165:427-31.

Pakkenberg B, Gundersen HJG (1997). Neocortical neuron number in humans: effect of sex and age. J Comp Neurol 384:312-20.

Small JV (1968). Measurement of section thickness. Fourth European Conference on Electron Microscopy, 609-10.

Sterio DC (1984). The unbiased estimation of number and sizes of arbitrary particles using the disector. J Microsc 134:127-36.

Tang Y, Nyengaard JR (1997). An unbiased stereological method for estimating the total length and the size distribution of the myelin fibre in human brain white matter. J Neurosci Meth 73:193-200.

Tang Y, Nyengaard JR, Pakkenberg B, Gundersen HJG (1998). Age-induced white matter changes in the human brain: a stereological investigation. Neurobiol Aging 18 (6):609-15.

Tang Y, Nyengaard JR, De Groot DMG, Gundersen HJG (2001). Total regional and global number of synapses in the human brain neocortex. SYNAPSE 41:258-73. 